\title{
Two-Dimensional Solid-State NMR Reveals Two Topologies of Sarcolipin in Oriented Lipid Bilayers ${ }^{\dagger}$
}

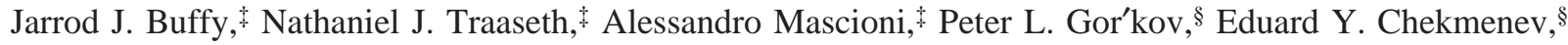 \\ William W. Brey, ${ }^{\S}$ and Gianluigi Veglia*,\$ \\ Department of Chemistry and Department of Biochemistry, Molecular Biology, and Biophysics, University of Minnesota, \\ Minneapolis, Minnesota 55455, and National High Magnetic Field Laboratory, Florida State University, \\ Tallahassee, Florida 32310
}

Received April 14, 2006; Revised Manuscript Received May 17, 2006

\begin{abstract}
Sarcolipin (SLN), a 31 amino acid integral membrane protein, regulates SERCA1a and SERCA2a, two isoforms of the sarco(endo)plasmic Ca-ATPase, by lowering their apparent $\mathrm{Ca}^{2+}$ affinity and thereby enabling muscle relaxation. SLN is expressed in both fast-twitch and slow-twitch muscle fibers with significant expression levels also found in the cardiac muscle. SLN shares $\sim 30 \%$ identity with the transmembrane domain of phospholamban (PLN), and recent solution NMR studies carried out in detergent micelles indicate that the two polypeptides bind to SERCA in a similar manner. Previous 1D solid-state NMR experiments on selectively ${ }^{15} \mathrm{~N}$-labeled sites showed that SLN crosses the lipid bilayer with an orientation nearly parallel to the bilayer normal. With a view toward the characterization of SLN structure and its interactions with both lipids and SERCA, herein we report our initial structural and topological assignments of SLN in mechanically oriented DOPC/DOPE lipid bilayers as mapped by 2D ${ }^{15} \mathrm{~N}$ PISEMA experiments. The PISEMA spectra obtained on uniformly ${ }^{15} \mathrm{~N}$-labeled protein as well as ${ }^{15} \mathrm{~N}-\mathrm{Leu},{ }^{15} \mathrm{~N}$-Ile and ${ }^{15} \mathrm{~N}-\mathrm{Val}$ map the secondary structure of SLN and, simultaneously, reveal that SLN exists in two distinct topologies. Both the major and the minor populations assume an orientation with the helix axis tilted by $\sim 23^{\circ}$ with respect to the lipid bilayer normal, but vary in the rotation angle about the helix axis by $\sim 5^{\circ}$. The existence of the multiple populations in model membranes may be a significant requirement for SLN interaction with SERCA.
\end{abstract}

The integral membrane protein sarcolipin (SLN) is expressed in both fast-twitch and slow-twitch muscle fibers. Recently, significant expression levels of SLN have been identified in cardiac muscle, increasing interest in this protein. In rats and mice, SLN expression is restricted to the heart and skeletal muscle, whereas in humans and rabbits, a different expression pattern is observed $(1-4)$. The different localization pattern amongst species has been attributed to sequence variations in the $\mathrm{N}$-terminal and/or transmembrane residues of SLN. MacLennan and co-workers found that the highly conserved $C$-terminal tail (RSYQY) of SLN is responsible for the localization of SLN in the endoplasmic reticulum (5).

The specific role of SLN in the regulation of heart muscle has yet to be fully understood. One hypothesis is that SLN increases the rate of the leakage of $\mathrm{Ca}^{2+}$ into the lumen,

$\dagger$ This work was supported by the National Institutes of Health Grant GM64742 and K02HL080081 to Gianluigi Veglia. J.J.B. was supported by the Minnesota Craniofacial Research Training Program (MinnCResT) and the NIH National Institute of Dental and Craniofacial Research (NIDCR 5T32-DE007288-10), and N.J.T. was supported by an American Heart Association Greater Midwest Affiliate pre-doctoral fellowship (0515491Z). The spectroscopy was performed at the NHMFL supported by NSF through cooperative agreement DMR-0084173 and by the State of Florida.

* Corresponding author. Tel: (612) 625-0758. Fax: (612) 626-7541. E-mail: veglia@chem.umn.edu.

University of Minnesota.

$\S$ Florida State University. augmenting ATP hydrolysis mediated heat production (6, 7). A second hypothesis is that SLN physically interacts with SERCA2a in tandem with phospholamban (PLN), its functional homolog, constituting a superinhibitory complex (8). In support of the latter hypothesis, in vivo and in vitro experiments show that SLN alone lowers SERCA's apparent $\mathrm{Ca}^{2+}$ affinity in a manner similar to that by PLN (9), whereas the simultaneous reconstitution of SLN and PLN produces a superinhibitory effect on SERCA (8).

Initially, no posttranslational modifications were identified in SLN co-purified with SERCA (10), leading to the hypothesis that its variable expression levels would be responsible for its regulation (2). Recently, however, the role of SLN in cardiac physiology was investigated using transgenic mouse models $(11,12)$. Specifically, it was found that the overexpression of SLN affects calcium transport on the sarco(endo)plasmic reticulum, decreasing the amplitude of the calcium transient and the rate of muscle relaxation (11). Although these studies exclude the ancillary role of SLN and its combined superinhibitory activity with PLN, they proposed that SLN is a novel regulator of SERCA, postulating that the inhibitory effect of SLN can be reversed by $\beta$-adrenergic stimulation. Additional studies found that, when expressed in PLN knock-out mice, SLN is able to regulate SERCA and that SLN inhibition can be fully reversed by treatments with isoproterenol (13). This suggests that the SLN inhibition of SERCA is regulated via a 
phosphorylation mechanism, analogous to that of PLN. Finally, it was shown that SLN can be phosphorylated at Thr5 when cotransfected with serine-threonine kinase 16 (STK16), making SLN as important as PLN for cardiac muscle relaxation (13).

Our previous solution NMR studies, carried out in sodium dodecyl sulfate (SDS) micelles, showed that the 22 transmembrane amino acids of SLN adopt a helical conformation and contain two short unstructured termini consisting of residues $1-6$ in the $N$-terminus and residues $27-31$ in the $C$-terminus $(14,15)$. More recently, we have solved the SLN structure in dodecyl phosphocholine (DPC) micelles, showing that in both SDS and DPC this protein adopts the same conformation (14). In addition, spin relaxation measurements carried out in DPC micelles partitioned SLN into four dynamic subdomains: a short unstructured $N$-terminus (residues 1-6), a short dynamic helix (residues 7-14), a more rigid helix (residues 15-26), and an unstructured $C$-terminus (residues 27-31). This dynamic nature of SLN is key to understanding its regulatory function of SERCA. In fact, our binding studies show that upon interacting with SERCA the different subdomains of SLN behave according to their dynamics, analogous to the transmembrane domain of PLN (TM-PLN) $(14,15)$. This result underscores the high sequence homology between SLN and TM-PLN, which share $\sim 30 \%$ identity (16). Because the structure, dynamics, and function of SLN are very similar to those of TM-PLN, we proposed that both SLN and PLN act using a similar mechanism by binding to the same groove on SERCA (14). Our conclusions are in agreement with mutagenesis studies, cross-linking experiments, and computational modeling carried out by MacLennan and co-workers (13).

Most of our previous structural, dynamics, and interaction studies were carried out in the isotropic environment of micelles. Conversely, in vivo SLN regulates SERCA in lipid membranes through intramembrane protein - protein interactions, where the relative orientations of both SLN and SERCA within the lipid membrane are crucially important for understanding the molecular mechanism for the SERCA/ SLN complex formation. In this work, we employ 2D solidstate NMR polarization-inversion spin exchange at the magic angle (PISEMA) $(17,18)$ to map the secondary structure of SLN and reveal that SLN exists in two distinct topologies. Both the major and the minor populations assume an orientation with the helix axis tilted by $\sim 23^{\circ}$ with respect to the lipid bilayer normal, but vary in the rotation angle about the helix axis by $\sim 5^{\circ}$. This elucidation of the SLN structure and topology in mechanically oriented lipid bilayers is a propaedeutic step toward the characterization of the SERCA/PLN complex.

\section{MATERIAL AND METHODS}

Protein Production and Reconstitution. 1,2-Dioleoyl-snglycero-3-phosphocholine (DOPC) and 1,2-dioleoyl-snglycero-3-phosphoethanolamine (DOPE) were purchased from Avanti Polar Lipids (Alabaster, AL). ${ }^{15} \mathrm{~N}$-labeled SLN was expressed according to the previously reported protocol (19). Mechanically aligned lipid bilayer samples were prepared in a manner similar to the previously published procedure (18). Briefly, DOPC/DOPE (4:1) was suspended in distilled water and sonicated using a Branson Sonifier until the solution was nearly transparent. Next, SLN was added to the lipid suspension (200:1 L/P), deposited on thin glass plates, dehydrated, and then slowly rehydrated (95\% RH) at a temperature above the main-phase transition temperature of the lipid mixture. Finally, the samples were sealed to prevent dehydration during data acquisition.

Solid State NMR Spectroscopy. NMR experiments were performed on a Bruker DMX-600 (National High Magnetic Field Laboratory, Tallahassee, FL) operating at a frequency of $600.14 \mathrm{MHz}$ for ${ }^{1} \mathrm{H}$ and $60.82 \mathrm{MHz}$ for ${ }^{15} \mathrm{~N}$. Prior to ${ }^{15} \mathrm{~N}$ data acquisition, the integrity of the oriented lipid bilayer was verified using ${ }^{31} \mathrm{P}$ NMR. The PISEMA experiments (17) were performed on a probe designed and constructed at the National High Magnetic Field Laboratory. To reduce sample heating, the probe utilizes a separate, low inductance ${ }^{1} \mathrm{H}$ resonator outside the ${ }^{15} \mathrm{~N}$ detection coil. Typical NMR parameters used in the PISEMA experiments include a ${ }^{1} \mathrm{H}$ pulse length of $3.7 \mu \mathrm{s}$, decoupling field of $48 \mathrm{kHz}$, rf field strength of $67.6 \mathrm{kHz}$ for ${ }^{1} \mathrm{H}$ during the SEMA element, and a recycle delay of $3.5-5 \mathrm{~s}$. The PISEMA spectra on selectively ${ }^{15} \mathrm{~N}$-labeled SLN were acquired at $5{ }^{\circ} \mathrm{C}$ with $9-12$ $t_{1}$ increments and $3 \mathrm{k}$ scans, whereas uniformly ${ }^{15} \mathrm{~N}$-labeled SLN samples required $32 t_{1}$ increments and $2 \mathrm{k}$ scans.

PISEMA Spectra Assignment and Simulations. The assignments of the peaks in the PISEMA spectra were obtained by an in-house automated assignment program. The program uses an exhaustive search algorithm to fit a list of resonances from the PISEMA spectra and simultaneously derives resonance assignments, the tilt angle $(\theta)$ of the helix relative to the direction of $\mathbf{B}_{0}$, and the rotation angle $(\rho)$ around the helix axis. These angular parameters are back-calculated by fitting the experimental values of dipolar coupling (DC) and chemical shift anisotropy (CSA) through a newly defined mathematical expression, which is a function of the secondary structure orientation and residue number $(20,21)$. Note that a similar mathematical treatment was used by Mascioni and Veglia for the interpretation of the regular pattern described by residual dipolar couplings (20). The DC can be expressed as

$$
D=A^{I} \sin \left(2 \alpha+\Psi^{I}\right)+A^{I I} \sin \left(\alpha+\Psi^{I I}\right)+A v
$$

and the CSA can be formulated as

$$
\sigma_{i}=\sum_{i=1,2,3} A_{i}^{I} \sin \left(2 \alpha+\Psi_{i}^{I}\right)+A_{i}^{I I} \sin \left(\alpha+\Psi_{i}^{I I}\right)+A v_{i}
$$

The expressions for the coefficients and the definitions for all of the variables are reported in Table 1. The full mathematical treatment will be reported elsewhere.

The algorithm for fitting the PISEMA pattern is similar to that reported by Opella and co-workers $(22,23)$ and performs the assignment in two stages using two peaks lists as an input. The first list contains the values of CSA and DC obtained from the peak peaking of the PISEMA spectrum of the uniformly labeled protein. The second list contains CSA and DC values from one selectively ${ }^{15} \mathrm{~N}$-labeled sample. In the first stage, the program derives the assignment for the selectively labeled sample, whereas in the second stage, the assigned peaks are used to derive the assignment for the rest of the residues in the uniformly labeled spectrum. During the first stage, the assignment is unknown, and the peaks are entered in the input files in a random order. The algorithm 


\section{Table 1}

dipolar couplings ${ }^{a}$

$$
\begin{aligned}
& A^{I}=\frac{3}{2} K_{D D} \sin ^{2} \theta \sin ^{2} \delta \\
& A^{I I}=-6 K_{D D} \sin \theta \cos \theta \sin \delta \cos \delta \\
& A v=\frac{3}{2} K_{D D} \sin ^{2} \theta \sin ^{2} \delta+3 K_{D D} \cos ^{2} \theta \cos ^{2} \delta-1 \\
& \Psi^{I}=2 \rho+\frac{\pi}{2} \\
& \Psi^{I I}=\rho+\frac{\pi}{2}
\end{aligned}
$$

chemical shift anisotropy ${ }^{b}$

$$
\begin{aligned}
& A_{i}^{I}=\frac{1}{2} \sigma_{i i} \sin ^{2} \theta \sin ^{2} \delta_{i i} \\
& A_{i}^{I I}=-2 \sigma_{i i} \sin \theta \cos \theta \sin \delta_{i i} \cos \delta_{i i} \\
& A v_{i}=\frac{1}{2} \sigma_{i i} \sin ^{2} \theta \sin ^{2} \delta_{i i}+\sigma_{i i} \cos ^{2} \theta \cos ^{2} \delta_{i i} \\
& \Psi^{I}{ }_{i}=2 \rho_{i}+\frac{\pi}{2} \\
& \Psi^{I I}{ }_{i}=\rho_{i}+\frac{\pi}{2}
\end{aligned}
$$

${ }^{a} K_{\mathrm{DD}}$, dipolar coupling constant; $\theta$, tilt angle of the helix axis with respect to the normal to the membrane bilayer; $\delta$, angle between the NH vector and the helix axis; $\rho$, rotation angle of the helix around its axis. ${ }^{b} \sigma_{i i}$, component $i$ of the CSA tensor; $\delta_{i i}$, angle between component $i$ of the CSA tensor and the helix axis; $\rho_{i}$, rotation angle of component $i$ of the CSA tensor around the helix axis.

derives an approximate tilt angle $\theta$ from the amplitude and position of the wheel on the PISEMA spectra. This is accomplished by fitting the maximum and minimum values of the experimental CSA and dipolar coupling from the list of resonances in the uniformly labeled spectrum and calculating the values of $\theta$ and $\rho$ corresponding to the best value of the score function

$$
\begin{aligned}
& \text { Score }= \\
& \begin{array}{r}
\sqrt{\left(D D_{\text {th }}^{\max }-D D_{\exp }^{\max }\right)^{2}+\left(D D_{\text {th }}^{\min }-D D_{\exp }^{\min }\right)^{2}} \cdot \frac{C S A_{\exp }^{\max }}{D D_{\exp }^{\max }}+ \\
\sqrt{\left(C S A_{\mathrm{th}}^{\max }-C S A_{\mathrm{exp}}^{\max }\right)^{2}+\left(C S A_{\mathrm{th}}^{\min }-C S A_{\exp }^{\min }\right)^{2}}
\end{array}
\end{aligned}
$$

where $D D_{\text {th }}^{\max }, D D_{\text {exp }}^{\max }, D D_{\text {th }}^{\min }, D D_{\text {exp }}^{\min }, C S A_{\text {th }}^{\max }, C S A_{\text {exp }}^{\max }$, $C S A_{\text {th }}^{\min }, C S A_{\text {exp }}^{\min }$ are the minimum and maximum values of the experimental and theoretical dipolar couplings and chemical shift anisotropy, respectively. The theoretical values of $D D_{\mathrm{th}}^{\max }$ and $C S A_{\mathrm{th}}^{\max }$ are calculated mathematically for each value of $\theta$ in the range $0-180^{\circ}$ and $\rho$ from $0-360^{\circ}$. The normalization factor $C S A_{\exp }^{\max } / D D_{\exp }^{\max }$ is used to take into account the differences in the orders of magnitude between the dipolar coupling and the chemical shift anisotropy. The calculation can be automatically repeated for different values of $\delta$ (the angle that the NH vector makes with the helix axis) and $T$ (the helix periodicity) using the range and step-size provided by the user. The initial values for these parameters are generally chosen to be $\delta \sim 17^{\circ}$ and $T=3.6$, values representative of an ideal $\alpha$-helix.

The value obtained for $\theta$ is then used to fit the frequencies from the selectively labeled spectrum and derive their assignments. As with the uniformly labeled sample, the frequencies are entered in the peak list in a random order. Because the residues that have been selectively labeled are not necessarily adjacent, the peaks must be entered into the list with the number corresponding to the sequence number that they have in the primary sequence. At this point, the algorithm predicts the assignment through a permutation of the peak order in the peak list, while keeping the distances fixed between residue pairs according to the primary sequence, until all of the possible permutations are tested. There is no need to know any of the assignments a priori. For each permutation, a theoretical PISA wheel is back- calculated using the previously determined value of $\theta$ and changing the value of $\rho$ to minimize the following score function

$$
\begin{aligned}
\text { Score }=\sqrt{\left(D D_{i}^{\exp }-D D_{j}^{\mathrm{th}}\right)^{2}} \cdot \frac{C S A_{\text {max }}^{\exp }}{D D_{\max }^{\exp }}+ & \\
& \sqrt{\left(C S A_{i}^{\exp }-C S A_{j}^{\mathrm{th}}\right)^{2}}
\end{aligned}
$$

where $i$ and $j$ denote the peak number, and $D D_{i}^{\exp }, D D_{j}^{\text {th }}$, $C S A_{i}^{\text {exp }}, C S A_{j}^{\text {th }}$ are the corresponding values of experimental and theoretical dipolar couplings and chemical shift anisotropies. $D D_{\exp }^{\max }, C S A_{\exp }^{\max }$ are the maximum values of the experimental dipolar coupling and chemical shift anisotropy.

The permutations of the resonances are generated by aligning the first theoretical peak with the first experimental peak through a rotation of the angle $\rho$ and then permuting the order of the remaining experimental peaks in the peak list. The process is repeated for the remaining peaks by aligning the first theoretical peak with each subsequent experimental point in the peak list. The solution with the lower score is taken as the assignment of the selectively labeled PISA wheel.

In the second stage, the selectively labeled peaks that are assigned are used to assign the uniformly labeled spectrum. The peaks on the uniformly labeled spectrum that correspond to those residues that have been assigned by the selectively labeled data are rearranged in the order provided by the algorithm, obtaining a partially assigned peak list. In this way, the position of the peaks along the PISA wheel trajectory is synchronized with the newly assigned peaks. The permutation algorithm is then run a second time using the updated peak list, leading to the assignment of the remaining peaks of the uniformly labeled PISA wheel. The process is repeated independently for each PISA wheel (corresponding to each individual helical fragment) identified on the spectra.

\section{RESULTS}

Assignment of the Oriented Spectra of SLN. SERCA has shown maximal activity when reconstituted in a 4:1 ratio of DOPC/DOPE lipid mixture; hence we carried out our SLN 

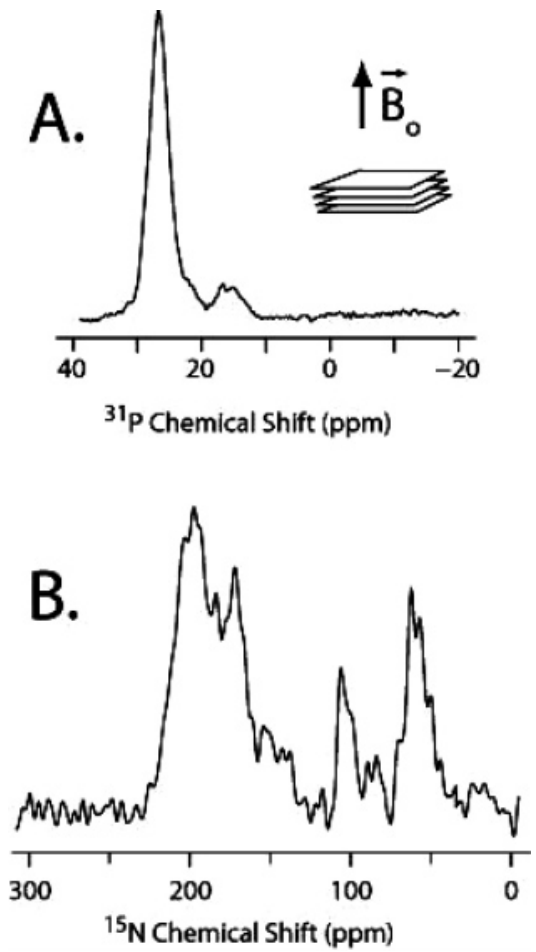

FIGURE 1: (A) Representative 1D ${ }^{31} \mathrm{P}$ spectrum of DOPC/DOPE oriented lipid bilayer in the presence of SLN. The presence of the peak near $30 \mathrm{ppm}$ in (A) indicates that the lipids are well oriented. (B) $1 \mathrm{D}^{15} \mathrm{~N}$ spectra of SLN in mechanically aligned lipid bilayers. The envelope of peaks resonating between 200 and $65 \mathrm{ppm}$ represents the portion of SLN that is oriented parallel and perpendicular to the magnetic field, respectively, whereas the peak near $120 \mathrm{ppm}$ represents the mobile, isotropic region of SLN.

structural analysis under these experimental conditions. Figure 1A shows a representative $1 \mathrm{D}{ }^{31} \mathrm{P}$ spectrum of a sample containing SLN reconstituted at a lipid-to-peptide ratio (L/P) of 200:1. The presence of a peak at $\sim 30 \mathrm{ppm}$ indicates that a uniform lipid bilayer has formed, whereas the presence of a small peak at $\sim 17 \mathrm{ppm}$ is likely due to the formation of DOPE rich domains (24). The corresponding ${ }^{15} \mathrm{~N}$ spectrum of uniformly ${ }^{15} \mathrm{~N}$-labeled SLN, Figure 1B, shows that SLN is well oriented in DOPC/DOPE as indicated by the peak near $200 \mathrm{ppm}$, which corresponds to the parallel component of the chemical shift tensor, relative to the magnetic field. The peak centered near $65 \mathrm{ppm}$ corresponds to a portion of SLN that is on the surface of the lipid bilayer, adopting an orientation perpendicular to the magnetic field. It is worth noting that SLN structural studies, in both DPC and SDS micelles, $(14,15)$, indicate that the putative transmembrane domain extends from $\sim$ Arg-6-Val-28, leaving the remaining $\sim 9$ residues outside of the bilayer.

Although the 1D spectrum can be used to obtain a semiquantitative orientation of SLN in lipid bilayers to unequivocally determine the topology of SLN, it is essential to correlate the CSA and DC, both of which have orientationdependent components (26), through 2D PISEMA NMR spectroscopy. The 2D PISEMA of uniformly ${ }^{15} \mathrm{~N}$-labeled SLN in oriented DOPC/DOPE lipid bilayers is shown in Figure 2. The main features of this spectrum are the presence of downfield peaks near 200 ppm, adopting a wheel-like pattern, as well as a smaller cluster of upfield peaks centered near $65 \mathrm{ppm}$. The downfield and upfield peaks correspond to amide vectors of SLN oriented parallel and perpendicular,

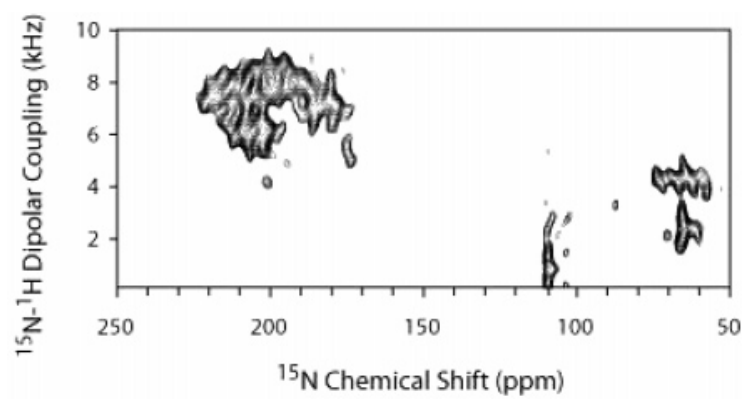

FIGURE 2: PISEMA of uniformly ${ }^{15} \mathrm{~N}$-labeled SLN in oriented lipid bilayers. A distinct PISA wheel pattern centered near $190 \mathrm{ppm}$ is indicative of a well-aligned helix, although complete site-specific resolution is not achieved at this $\mathrm{B}_{0}$ field.

respectively, relative to the static magnetic field. Although the samples are well aligned, the resonances appear broad, which necessitated the preparation of ${ }^{15} \mathrm{~N}-\mathrm{Leu},{ }^{15} \mathrm{~N}-\mathrm{Ile}$, and ${ }^{15} \mathrm{~N}-\mathrm{V}$ al samples to aid in the backbone assignment. The PISEMA spectra of ${ }^{15} \mathrm{~N}$-Leu (A), ${ }^{15} \mathrm{~N}-\mathrm{Ile}(\mathrm{B})$, and ${ }^{15} \mathrm{~N}-\mathrm{Val}$ (C) are shown in Figure 3. Unlike the resonances in the $\mathrm{U}^{15} \mathrm{~N}$ labeled spectrum, most of the individual resonances are discernible in the selectively labeled samples. Val-15, 19, and 26 are poorly resolved because they are located on the same face of the helix. The CSA and DC for each resonance were input as constraints into our search algorithm as described in Material and Methods. By fitting all of the residues simultaneously, we obtained $\theta=23 \pm 2^{\circ}$ relative to the bilayer normal and assigned all of the residues located in the helical portion of SLN. Our backbone assignment of SLN is supported by the specific pattern of each selectively labeled sample, giving a unique solution for the orientation of the helix $(27,28)$ and the previous 1D data collected on synthetic SLN (15).

Moreover, it should be noted that the peak intensities in the PISEMA spectra are not uniform. For instance, Ile-14 and Ile-17 have lower intensities than those of Ile-3 and Ile20 (Figure 3B). Ile-14 and Ile-17 are located near a helical kink, proposed from detergent micelle studies, which likely exhibits variable dynamics. It is interesting to note that Leu16 shows similarly low intensity relative to the other Leu residues. One possibility for the lower intensity is that the cross polarization element, which is sensitive to protein dynamics, is less efficient for certain dynamic regions of SLN. Another explanation comes from the recent analysis of the PISEMA peak intensities carried out by Cross and co-workers (28), who found that a change in the static magnetic field occurs for each single residue in the protein sequence and is a function of its position of the specific resonance in the PISEMA frequency plane. This in turn would affect the relative intensity of the resonances in the PISA wheel. As a result, the intensity may be attenuated because Ile-14 is positioned closer than the other residues to the so-called sweet spot, which denotes the minimum intensity point (28). Remarkably, Ile-3 falls in the oriented $\alpha$-helical region of the PISEMA spectrum, suggesting that the helical conformation extends up to this residue in the lipid bilayer.

In addition to the information regarding the tilt of the helix with respect to the lipid bilayer, the specific patterns observed for the selectively labeled samples reveal that the helical face comprising Leu-21 and Leu-25 points towards the surface 

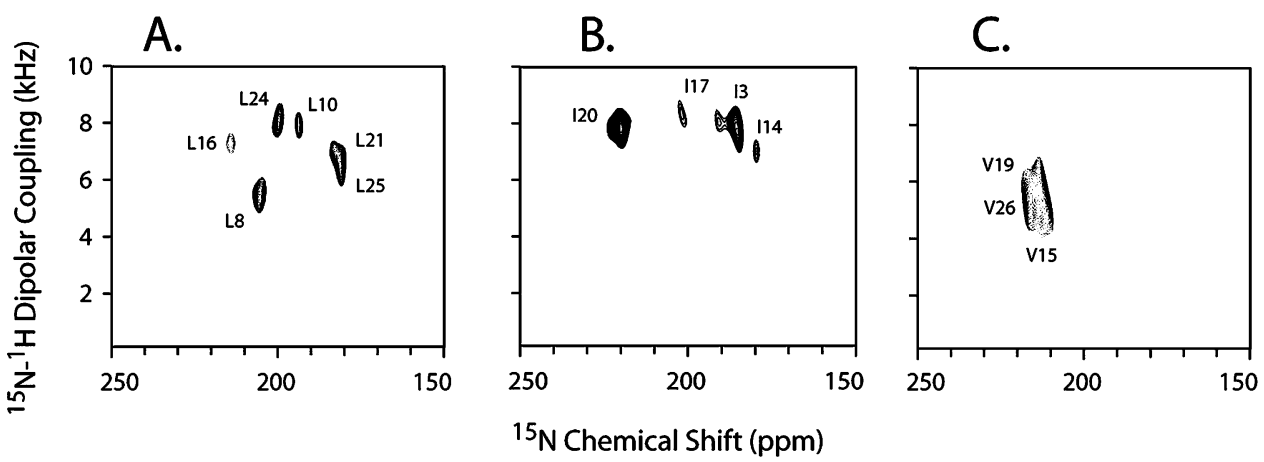

FIGURE 3: PISEMA spectra and assignment of (A) ${ }^{15} \mathrm{~N}$-Leu SLN, (B) ${ }^{15} \mathrm{~N}$-Ile SLN, and (C) ${ }^{15} \mathrm{~N}-\mathrm{Val}$ SLN in oriented lipid bilayers. Note that the three Val residues are nearly coincident on the helical wheels and are not completely resolved in the spectrum. The ${ }^{15} \mathrm{~N}-\mathrm{Leu}$, ${ }^{15} \mathrm{~N}$-Ile, and ${ }^{15} \mathrm{~N}$-Val spectra show wheel patterns that follow those seen for the uniformly ${ }^{15} \mathrm{~N}$-labeled SLN spectrum in Figure 2 . The asymmetric distribution of the resonances in the PISEMA spectrum indicates the rotation of the helical axis in the lipid bilayers.

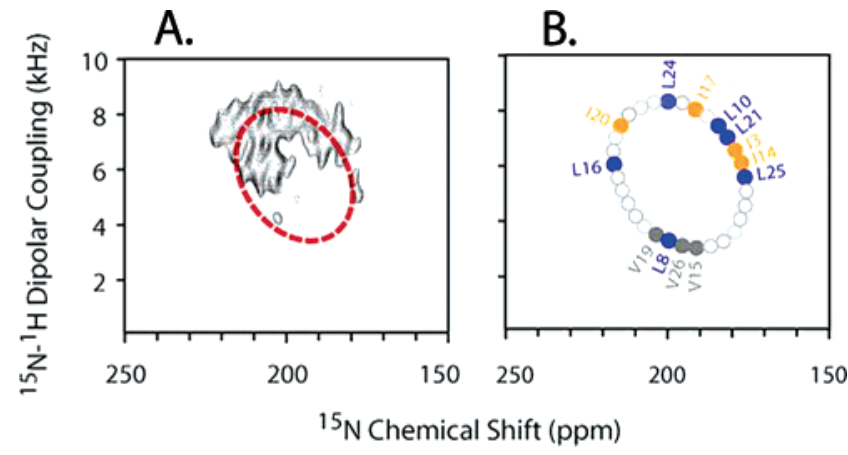

Figure 4: (A) Theoretical PISEMA wheel-pattern of polyalanine with a helical tilt $(\theta)$ of $23^{\circ}$ with respect to the bilayer normal, fit to uniformly ${ }^{15} \mathrm{~N}$-labeled SLN. (B) PISA wheel of SLN calculated from an ideal helix. The residues assigned are highlighted.
A.

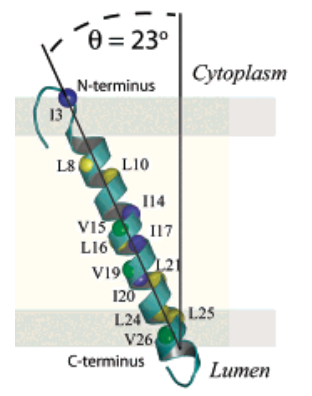

B.
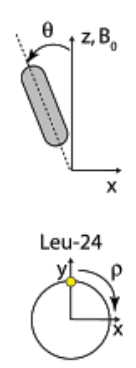

FIGURE 5: Proposed structural model of SLN in lipid membranes. (A) The Leu, Ile and Val residues that are assigned in the PISEMA spectra are highlighted in yellow, blue and green spheres, respectively. SLN backbone is rotated around its helical axis so that the face containing Leu-21, Leu-25, and Ile-14 points toward the $\mathrm{N}$-terminal side of the membrane, corresponding to the cytoplasm side in SR (5). (B) Definition of $\theta$ and $\rho$ angles.

of the oriented lipid bilayer. In particular, ${ }^{15} \mathrm{~N}$-Leu PISEMA shows that these leucine residues possess ${ }^{1} \mathrm{H}_{-}{ }^{15} \mathrm{~N}$ dipolar couplings values that are indicative of the leucine $\mathrm{NH}$ vectors oriented almost parallel to the membrane normal. The fit of our experimental ${ }^{15} \mathrm{~N}$-SLN PISEMA (A) and our PISA wheel assignment (B) are shown in Figure 4. According to our fit and assignment of our PISEMA spectra, and the implication that the conserved $C$-terminal tail (RSYQY) of SLN is directed towards the lumen (5), our current model of SLN topology in oriented DOPC/DOPE is shown in Figure 5.

Two Topologies of SLN in Oriented Lipid Bilayers. Given the high degree of alignment obtained with the SLN samples the resolution achieved for each amide site in the PISEMA

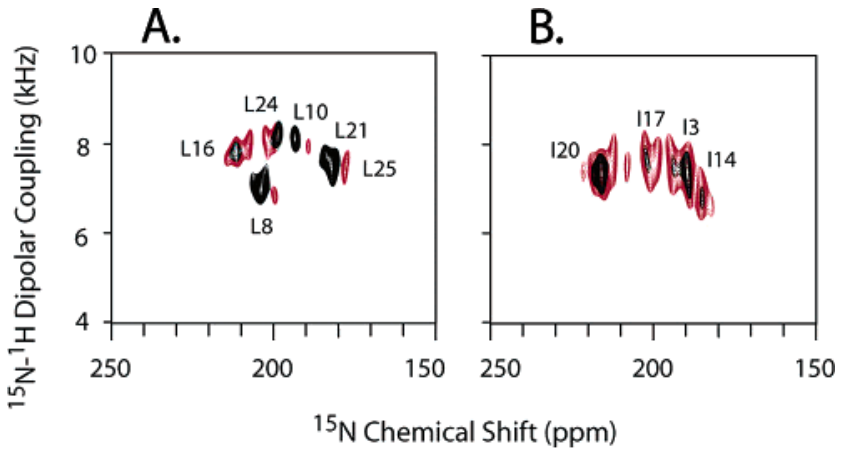

FIGURE 6: PISEMA spectrum of (A) ${ }^{15} \mathrm{~N}-$ Leu and (B) ${ }^{15} \mathrm{~N}-\mathrm{Ile}$ SLN in oriented DOPC/DOPE showing the additional topological conformer shown in red.

spectrum for $\mathrm{U}^{15} \mathrm{~N}$ labeled protein is rather poor. In general, the reduction in spectral resolution can be caused by nonhomogeneous sample preparations or by intrinsic protein dynamics, resulting in a Gaussian broadening of the spectral lines (28). For SLN, the inspection of lower contour levels of spectra from PISEMA experiments reveals the presence of an additional set of discrete peaks. In particular, a close analysis of ${ }^{15} \mathrm{~N}$-Leu- and ${ }^{15} \mathrm{~N}$-Ile-labeled SLN spectra reveal the presence of peak doubling. For example, Figure 6 shows the upper quadrant of the PISEMA spectrum for the (A) ${ }^{15} \mathrm{~N}$ Leu and (B) ${ }^{15} \mathrm{~N}$-Ile spectra plotted at a threshold lower than that in Figure 3. In addition to the major peaks observed in Figure 3 for each residue, this spectrum shows an additional set of peaks, equal to the number of observable Leu peaks, with $\sim 60 \%$ of the intensity of the principal population. Because both HSQC spectra and mass spectrometry analysis carried out on these protein preparations indicate that no detectable label scrambling has occurred during protein biosynthesis, these peaks can be assigned to two different topologies of SLN in DOPC/DOPE lipid bilayers. Our structural fitting shows that with respect to the major population the second population is rotated by $\sim 5^{\circ}$ about its helical axis $(\rho)$ and with a change in $\theta$ of $\sim 2^{\circ}$. The PISEMA experiments carried out as function of temperature as well as with SLN reconstituted using a different lipid composition demonstrate that there is a slow equilibrium between the two topologies (manuscript in preparation). Note that to be detectable on the NMR time scale, this topological interconversion must occur on a time scale that is slower than $10^{-4} \mathrm{~s}$, which would not completely average the chemical shift (25). Interestingly, the two populations are also present 
A.

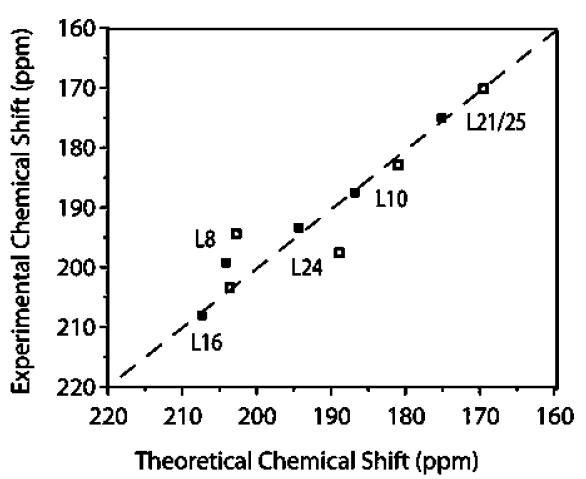

B.

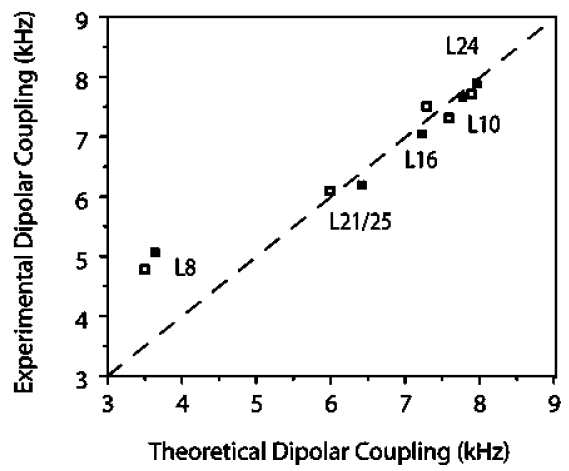

FIGURE 7: Correlation plots for both (A) CSA and (B) DC showing the remarkable correlations for experimental ${ }^{15} \mathrm{~N}$-Leu values with the theoretical values of an ideal helix tilted by $23^{\circ}$ with respect to the membrane normal. The filled ( $\square$ ) and open squares ( $\square$ ) correspond to the major and minor populations, respectively.

in TM-PLN, showing that primary sequence conservation is connected to the conservation of protein dynamics (29).

Remarkably, for both populations, the experimental values of CSA and DC, measured for SLN residues from the PISEMA spectra, show good correlation with the theoretical values for an ideal polyalanine helix, tilted by $23 \pm 2^{\circ}$. The correlation plots for both CSA and DC are shown in Figure 7. The small discrepancies between the theoretical and experimental values of DC and CSA observed in Figure 7 for Leu-8 may be due to dynamics or the deviation from ideality. According to the structural dynamics of SLN in micelles, this residue exhibits a large amplitude motion that can be the cause for the scaled value of dipolar coupling and chemical shift anisotropy.

For both helical and beta-barrel membrane embedded proteins, the presence of slow dynamics and multiple populations detectable by PISEMA experiments was predicted by molecular dynamics calculations $(30,31)$. The resolution achieved in our 2D PISEMA experiments allowed us to exemplify and characterize multiple populations for the first time (Figure 5).

\section{DISCUSSION}

SLN adopts a nearly identical structure in both DPC and SDS micelles, with a 22 amino acid transmembrane helix (from residues Arg-6 through Arg-28) flanked by unstructured termini $(14,15)$. On the basis of these solution structures, 22 residues would be expected to resonate in the downfield region of the ${ }^{15} \mathrm{~N}$ CSA dimension of the PISEMA spectrum, whereas the remaining nine residues would be expected to be scaled by motion and resonate near the isotropic region of $\sim 115 \mathrm{ppm}$. Our data are in agreement with this prediction, showing that the residues in the helical conformation cluster around $\sim 200 \mathrm{ppm}$, a position indicative of a parallel orientation of the amide $\mathrm{N}-\mathrm{H}$ vector with respect to the magnetic field. The structural fitting carried out with our algorithm (see Material and Methods) identifies an angle of $23 \pm 2^{\circ}$ relative to the bilayer normal, which mirrors the conclusions of a similar investigation carried out with PLN under identical experimental conditions (29). This result demonstrates that highly homologous primary sequences (such as SLN and TM-PLN) are likely to have the same interactions with lipid bilayers.

The majority of the residues assigned have DC and CSA values in remarkable agreement with those of an ideal helix, with only a few residues deviating from ideality. With the support of the H/D exchange experiments (14), we can conclude that the helix portion of SLN embedded in the hydrocarbon region of the membrane has a helical conformation close to ideality because of the presence of strong hydrogen bonds, a conclusion that supports the theories of Cross and Engelmann groups $(32,33)$ about the ideality of transmembrane domains. Remarkably, the Ile-3 peak resonates in the region of the PISEMA spectrum that indicates a helix oriented $\sim 23 \pm 2^{\circ}$ relative to the bilayer normal because this region exhibits significant dynamics according to solution NMR studies in detergent micelles $(14,15)$. The resonance position of Ile-3 in the PISEMA spectrum shows that in lipid bilayer the helical structure of SLN may extend further than in detergent micelles. Contrary to our predictions based on solution NMR studies of SLN in detergent micelles, only one peak is present near the isotropic resonance position of $\sim 115 \mathrm{ppm}$. However, it is apparent that several residues interact with the lipid bilayer, as demonstrated by the weak but significant dipolar couplings observed for $\sim 8$ peaks clustered around $\sim 65 \mathrm{ppm}$. These differences between detergent micelles and lipid bilayers are due to the limitations imposed by the micellar systems that mimic the hydrocarbon region of the cell membrane relatively well but fall short in reproducing the lipid-water interfaces. In the case of SLN, the characterization of the membrane-water interface regions is of particular interest because the $C$-terminal residues contain the RSYQY sarco(endo)plasmic membrane localization sequence (5). The complete assignment of both backbone and side chain resonances in the lipid bilayer will be necessary to identify all of the residues at the membranewater interface.

An important aspect that emerges from the PISEMA experiments is the preferred orientation of SLN with respect to the membrane bilayer, with the face Leu-21 and Leu-25 pointing toward the bilayer surface. Remarkably, the PISEMA experiments under the conditions used detect the presence of a slow equilibrium between two topologies that interconvert on a slow time scale. These dynamics may play an important role in the intramembrane recognition of SLN by SERCA. In fact, our previous NMR studies on both PLB and SLN interactions with SERCA postulated the presence of a preexisting equilibrium between the $\mathrm{R}$ and the $\mathrm{T}$ states, more apparent in the dynamics of the cytoplasmic portion of PLN (34-36). Although SLN does not have a large cytoplasmic domain, spin relaxation measurements indicated that its transmembrane primary sequence encodes several dynamics from the picosecond to the millisecond time scales 
(36). These dynamics may be responsible for the topological interconversion of both SLN and PLN in lipids and be the driving force for the $\mathrm{R} \rightarrow \mathrm{T}$ transition. This in turn may constitute a preexisting equilibrium that facilitates the recognition by SERCA.

\section{CONCLUSIONS}

We used PISEMA spectra to map the structure and topology of SLN in oriented DOPC/DOPE bilayers. Under our experimental conditions, this single-pass membrane protein adopts a helical conformation and crosses the membrane with a tilt angle of $23 \pm 2^{\circ}$. PISEMA spectra obtained with both uniformly and selectively ${ }^{15} \mathrm{~N}$-labeled SLN clearly indicate a preferential orientation of the helix face with Leu-21, Leu-25, and Ile-14 pointing toward the bilayer surface. Remarkably, the PISEMA spectra show the presence of a second population of resonances. This additional population roughly exhibits the same tilt angle $(\theta)$ of the SLN helix with respect to the membrane bilayer but a slightly different rotational angle $(\rho)$. These two topologies may represent the two states whose information is totally lost in the isotropic micellar environments and may represent a preequilibrium state of SLN that constitutes a requirement for SERCA recognition.

These studies stress the importance of a combined solution/ solid-state NMR approach to solve the structure, topology, and dynamics of membrane proteins.

\section{ACKNOWLEDGMENT}

We thank Dr. T. Cross, Dr. R. Fu, Roberto Di Fonzo, and Robin Rothenbuhler for many helpful discussions.

\section{REFERENCES}

1. Odermatt, A., Taschner, P. E., Scherer, S. W., Beatty, B., Khanna, V. K., Cornblath, D. R., Chaudhry, V., Yee, W. C., Schrank, B., Karpati, G., Breuning, M. H., Knoers, N., and MacLennan, D. H. (1997) Characterization of the gene encoding human sarcolipin (SLN), a proteolipid associated with SERCA1: absence of structural mutations in five patients with Brody disease, Genomics $45,541-553$.

2. Gayan-Ramirez, G., Vanzeir, L., Wuytack, F., and Decramer, M. (2000) Corticosteroids decrease mRNA levels of SERCA pumps, whereas they increase sarcolipin mRNA in the rat diaphragm, $J$. Physiol. 524, 387-397.

3. Tupling, A. R., Asahi, M., and MacLennan, D. H. (2002) Sarcolipin overexpression in rat slow twitch muscle inhibits sarcoplasmic reticulum $\mathrm{Ca} 2+$ uptake and impairs contractile function, J. Biol. Chem. 277, 44740-44746.

4. Vangheluwe, P., Schuermans, M., Zador, E., Waelkens, E., Raeymaekers, L., and Wuytack, F. (2005) Sarcolipin and phospholamban mRNA and protein expression in cardiac and skeletal muscle of different species, Biochem. J. 389, 151-159.

5. Gramolini, A. O., Kislinger, T., Asahi, M., Li, W., Emili, A., and MacLennan, D. H. (2004) Sarcolipin retention in the endoplasmic reticulum depends on its $C$-terminal RSYQY sequence and its interaction with sarco(endo)plasmic $\mathrm{Ca}(2+)$-ATPases, Proc. Natl. Acad. Sci. U.S.A. 101, 16807-16812.

6. Lee, A. G. (2002) A calcium pump made visible. Curr. Opin. Struct. Biol. 12, 547-554.

7. Smith, W. S., Broadbridge, R., East, J. M., and Lee, A. G. (2002) Sarcolipin uncouples hydrolysis of ATP from accumulation of $\mathrm{Ca} 2+$ by the $\mathrm{Ca} 2+-\mathrm{ATPase}$ of skeletal-muscle sarcoplasmic reticulum, Biochem. J. 361, 277-286.

8. MacLennan, D. H., Asahi, M., and Tupling, A. R. (2003) The regulation of SERCA-type pumps by phospholamban and sarcolipin, Ann. N.Y. Acad. Sci. 986, 472-480.
9. Odermatt, A., Becker, S., Khanna, V. K., Kurzydlowski, K., Leisner, E., Pette, D., and MacLennan, D. H. (1998) Sarcolipin regulates the activity of SERCA1, the fast-twitch skeletal muscle sarcoplasmic reticulum Ca2+-ATPase, J. Biol. Chem. 273, $12360-12369$

10. Wawrzynow, A., Theibert, J. L., Murphy, C., Jona, I., Martonosi, A., and Collins, J. H. (1992) Sarcolipin, the "proteolipid" of skeletal muscle sarcoplasmic reticulum, is a unique, amphipathic, 31-residue peptide, Arch. Biochem. Biophys. 298, 620-623.

11. Babu, G. J., Bhupathy, P., Petrashevskaya, N. N., Wang, H., Raman, S., Wheeler, D., Jagatheesan, G., Wieczorek, D., Schwartz, A., Janssen, P. M., Ziolo, M. T., and Periasamy, M. (2006) Targeted overexpression of sarcolipin in the mouse heart decreases sarcoplasmic reticulum calcium transport and cardiac contractility, J. Biol. Chem. 281, 3972-3979.

12. Babu, G. J., Zheng, Z., Natarajan, P., Wheeler, D., Janssen, P. M., and Periasamy, M. (2005) Overexpression of sarcolipin decreases myocyte contractility and calcium transient, Cardiovasc. Res. 65, 177-186.

13. Gramolini, A. O., Trivieri, M. G., Oudit, G. Y., Kislinger, T., Li, W., Patel, M. M., Emili, A., Kranias, E. G., Backx, P. H., and Maclennan, D. H. (2006) Cardiac-specific overexpression of sarcolipin in phospholamban null mice impairs myocyte function that is restored by phosphorylation, Proc. Natl. Acad. Sci. U.S.A. $103,2446-2451$

14. Buffy, J. J., Buck-Koehntop, B. A., Porcelli, F., Traaseth, N. J., Thomas, D. D., and Veglia, G. (2006) Defining the intramembrane binding mechanism of sarcolipin to calcium ATPase using solution NMR spectroscopy, J. Mol. Biol. 358, 420-429.

15. Mascioni, A., Karim, C., Barany, G., Thomas, D. D., and Veglia, G. (2002) Structure and orientation of sarcolipin in lipid environments, Biochemistry 41, 475-482.

16. Hellstern, S., Pegoraro, S., Karim, C. B., Lustig, A., Thomas, D. D., Moroder, L., and Engel, J. (2001) Sarcolipin, the shorter homologue of phospholamban, forms oligomeric structures in detergent micelles and in liposomes, J. Biol. Chem. 276, 3084530852 .

17. Wu, C. H., Ramamoorthy, A., and Opella, S. J. (1994) Threedimensional solid-state NMR experiment that correlates the chemical shift and dipolar coupling frequencies of two heteronuclei, J. Magn. Reson. 109, 270-272.

18. Marassi, F. M., Ramamoorthy, A., and Opella, S. J. (1997) Complete resolution of the solid-state NMR spectrum of a uniformly $15 \mathrm{~N}$-labeled membrane protein in phospholipid bilayers, Proc. Natl. Acad. Sci. U.S.A. 94, 8551-8556.

19. Buck, B., Zamoon, J., Kirby, T. L., DeSilva, T. M., Karim, C., Thomas, D., and Veglia, G. (2003) Overexpression, purification, and characterization of recombinant Ca-ATPase regulators for high-resolution solution and solid-state NMR studies, Protein Expression Purif. 30, 253-261.

20. Mascioni, A., and Veglia, G. (2003) Theoretical analysis of residual dipolar coupling patterns in regular secondary structures of proteins, J. Am. Chem. Soc. 125, 12520-12526.

21. Mascioni, A., Eggimann, B. L., and Veglia, G. (2004) Determination of helical membrane protein topology using residual dipolar couplings and exhaustive search algorithm: application to phospholamban, Chem. Phys. Lipids 132, 133-144.

22. Marassi, F. M., and Opella, S. J. (2000) A solid-state NMR index of helical membrane protein structure and topology, J. Magn. Reson., Communications 144, 150-155.

23. Nevzorov, A. A., and Opella, S. J. (2003) Structural fitting of PISEMA spectra of aligned proteins, J. Magn. Reson. 160, 3339.

24. Henzler Wildman, K. A., Lee, D. K., and Ramamoorthy, A. (2003) Mechanism of lipid bilayer disruption by the human antimicrobial peptide, LL-37, Biochemistry 42, 6545-6558.

25. Abragam, A. (1961) Principles of Nuclear Magnetism, p 315 , Oxford University Press, New York.

26. Denny, J. K., Wang, J., Cross, T. A., and Quine, J. R. (2001) PISEMA powder patterns and PISA wheels, J. Magn. Reson. 152, $217-226$

27. Wang, J., Denny, J., Tian, C., Kim, S., Mo, Y., Kovacs, F. A., Song, Z., Nishimura, K., Gan, Z., Fu, R., Quine, J. R., and Cross, T. A. (2000) Imaging membrane protein helical wheels, J. Magn. Reson. 144, 162-167.

28. Quine, J. R., Achuthan, S., Asbury, T., Bertram, R., Chapman, M. S., Hu, J., and Cross, T. A. (2006) Intensity and mosaic spread analysis from PISEMA tensors in solid-state NMR, J. Magn. Reson. 179, 190-198. 
29. Traaseth, N. J., Buffy, J. J., Zamoon, J., and Veglia, G. (2006) Structural dynamics and topology of phospholamban in oriented lipid bilayers using multidimensional solid-state NMR spectroscopy, Biochemistry, submitted for publication.

30. Straus, S. K., Scott, W. R., and Watts, A. (2003) Assessing the effects of time and spatial averaging in $15 \mathrm{~N}$ chemical shift $/ 15 \mathrm{~N}$ $1 \mathrm{H}$ dipolar correlation solid-state NMR experiments, J. Biomol. NMR 26, 283-295.

31. Bleile, D. W., Scott, W. R., and Straus, S. K. (2005) Can PISEMA experiments be used to extract structural parameters for mobile beta-barrels? J. Biomol. NMR 32, 101-111.

32. Kim, S., and Cross, T. A. (2002) Uniformity, ideality, and hydrogen bonds in transmembrane alpha-helices, Biophys. J. 83, 2084-2095.

33. Senes, A., Ubarretxena-Belandia, I., and Engelman, D. M. (2001) The Calpha - - $\mathrm{H} \cdots \mathrm{O}$ hydrogen bond: a determinant of stability and specificity in transmembrane helix interactions, Proc. Natl. Acad. Sci. U.S.A. 98, 9056-9061.

34. Zamoon, J., Nitu, F., Karim, C., Thomas, D. D., and Veglia, G. (2005) Mapping the interaction surface of a membrane protein: unveiling the conformational switch of phospholamban in calcium pump regulation, Proc. Natl. Acad. Sci. U.S.A. 102, 47474752.

35. Traaseth, N. J., Thomas, D. D., and Veglia, G. (2006) Effects of Ser16 phosphorylation on the allosteric transitions of phospholamban/Ca2+-ATPase complex, J Mol Biol 358, 1041-1050.

36. Metcalfe, E. E., Zamoon, J., Thomas, D. D., and Veglia, G. (2004) ${ }^{1} \mathrm{H} /{ }^{15} \mathrm{~N}$ Heteronuclear NMR spectroscopy shows four dynamic domains for phospholamban reconstituted in dodecylphosphocholine micelles, Biophys. J. 87, 1-10.

BI060728D 